\title{
Endonasal Endoscopic Surgery in the Management of Sinonasal and Anterior Skull Base Malignancies
}

\author{
Christopher R. Roxbury ${ }^{1} \cdot$ Masaru Ishii ${ }^{1,2} \cdot J^{1}$ eremy D. Richmon ${ }^{1} \cdot$ \\ Ari M. Blitz ${ }^{3}$ Douglas D. Reh ${ }^{1,2} \cdot$ Gary L. Gallia ${ }^{1,2,4}$
}

Received: 26 August 2015/Accepted: 5 November 2015/Published online: 1 February 2016

(C) Springer Science+Business Media New York 2016

\begin{abstract}
Sinonasal malignancies represent a rare subset of tumors with a wide variety of histopathologic diagnoses and overall poor prognosis. These tumors tend to have an insidious onset with non-specific symptoms which often leads to delayed diagnosis and advanced local disease at presentation. The principal goal of surgery is to obtain a negative margin resection. Open craniofacial techniques are well established in the management of sinonasal malignancies and remain the treatment of choice for many advanced tumors. Over the past couple of decades, there has been tremendous application of endoscopic techniques to skull base pathologies including sinonasal malignancies. For selected cases, endonasal endoscopic techniques can be performed with curative intent and reduced surgical morbidity and mortality. Here we discuss principles of surgical management of sinonasal malignancies, review the techniques of endonasal endoscopic resection of sinonasal malignancies, and highlight the importance of pathology in the multi-disciplinary management of patients with these complex lesions.
\end{abstract}

Gary L. Gallia

ggallia1@jhmi.edu

1 Department of Otolaryngology-Head and Neck Surgery, Johns Hopkins University School of Medicine, Baltimore, MD 21287, USA

2 Department of Neurosurgery, Johns Hopkins University School of Medicine, 600 North Wolfe Street, Phipps Building, Room 101, Baltimore, MD 21287, USA

3 Division of Neuroradiology, Department of Radiology, Johns Hopkins University School of Medicine, Baltimore, MD 21287, USA

4 Department of Oncology, Johns Hopkins University School of Medicine, Baltimore, MD 21287, USA
Keywords Endoscopic - Expanded endonasal approach · Paranasal sinuses $\cdot$ Sinonasal malignancy $\cdot$ Skull base

\section{Introduction}

Sinonasal malignancies are a diverse group of uncommon tumors that represent challenging entities to treat. The overall incidence, based on the United States National Cancer Institute's Surveillance, Epidemiology, and End Results (SEER) registry is 0.83 per 100,000 people, with a slight male predominance [1]. These malignancies often have an insidious onset with non-specific symptoms including nasal obstruction, nasal discharge/epistaxis, facial pain, and headache. Given many of these symptoms are consistent with rhinosinusitis, early lesions are not commonly diagnosed. In advanced tumors, neurological deficits can develop. The average time from onset of symptoms to diagnosis is 6-12 months [2]. Despite advances in surgery, radiotherapy, and chemotherapy, prognosis for patients with sinonasal malignancies remains poor.

Malignancies involving the nose and paranasal sinuses include a varied group of histopathologic entities and this issue of Head and Neck Pathology is dedicated to many of these pathologies. In broad terms, these tumors can be categorized into epithelial, soft tissue tumors of bone and cartilage, haematolymphoid, neuroendocrine, germ cell, and secondary tumors; a complete listing of the classification of sinonasal malignancies has been provided by the World Health Organization [3]. Although there is some variability in different series, the most common malignancy involving the sinonasal cavity and anterior skull base is squamous cell carcinoma [4-6]. Given the numerous histopathological entities that can occur within 
the sinonasal cavity, it is not surprising that there are different treatment algorithms. Although the detailed algorithms are beyond the scope of this article and have been defined by the National Comprehensive Cancer Network [7], surgery followed by radiotherapy or a combination of chemotherapy and radiotherapy is considered for many patients.

Surgery has played and continues to play an important role in the management of sinonasal malignancies and techniques have changed over time. Craniofacial techniques, originally described in the 1960s [8], became the gold standard in the surgical management of malignancies involving the anterior skull base over the ensuing decades. These techniques remain the treatment of choice for many patients with advanced disease. In the 1990s, endoscopic techniques began to be applied to various skull base pathologies (Fig. 1). In the late 1990s and early 2000s, endoscopic approaches were applied to resection of sinonasal malignancies [9-21]. Current expanded endonasal endoscopic approaches provide excellent access to selected lesions involving the anterior, middle, and posterior cranial fossa and the craniocervical junction [22-25]. Here, we discuss general principles in the surgical management of sinonasal malignancies, review the technical aspects of the endonasal endoscopic approach and resection of sinonasal malignancies, and highlight the role of intraoperative margin assessment. As most of these malignancies involve the anterior cranial base [26], these will serve as the focus of this paper.

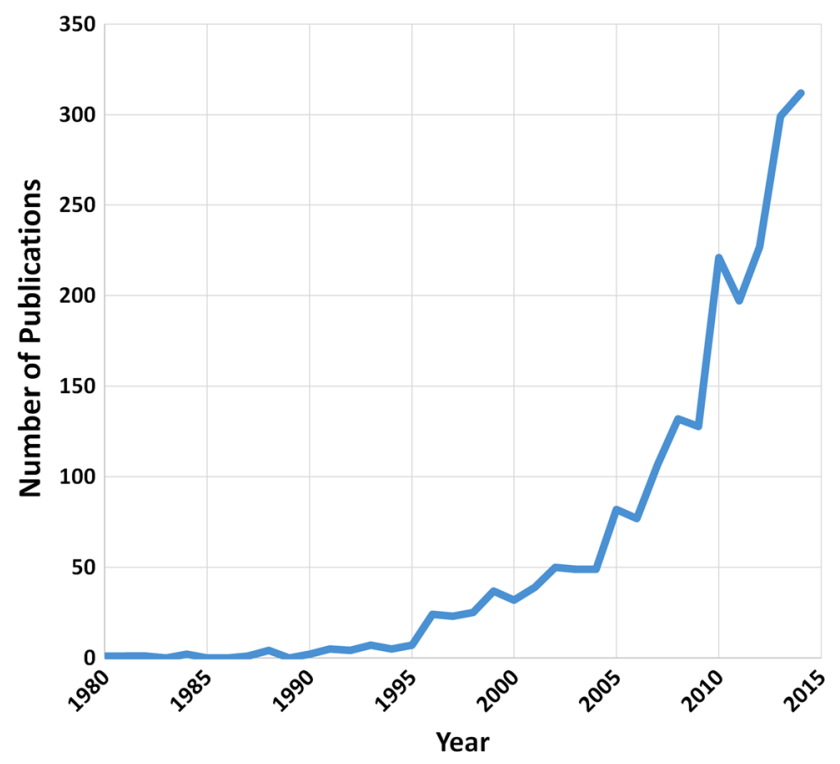

Fig. 1 Pubmed was accessed and searched for the terms "endoscopic skull base" on August 1, 2015. The number of publications is graphed per year from 1980 to 2014. Publication number began to increase between the years 1995-2000 with a dramatic increase noted over the past decade

\section{General Surgical Principles and Preoperative Evaluation}

The goals of surgical resection of anterior skull base malignancies are similar to those of oncologic surgery at other body sites. In general terms, the goal of surgery is to obtain a negative margin resection of the tumor. This must be achieved with as little morbidity as possible with preservation of critical anatomic structures where appropriate. In unresectable tumors, a palliative organ-sparing approach may be employed. Reconstruction is of particular importance in skull base surgery given the complexity of the anatomy and the need to separate the intracranial compartment from the sinonasal space and outside world.

Diagnostic evaluation and workup of suspected sinonasal malignancies should be performed by a multidisciplinary team with involvement of an otolaryngologist-head and neck surgeon, neurosurgeon, medical and radiation oncologists, neuroradiologist, and pathologist. Evaluation begins with a thorough examination of the head and neck. Neurologic examination should be performed with attention to signs of advanced disease such as cranial neuropathies. Nasal endoscopy is performed for determination of tumor origin and assessment of extent of disease, with attention also to how the tumor and anatomy affect access (e.g. septal deviation). In cases of tumors abutting and/or invading the orbit, ophthalmologic evaluation is considered.

Biopsy of a suspicious lesion for histopathologic diagnosis is performed for determination of surgical versus nonsurgical management and may be performed in the office setting or in the operating room should there be concern for potential epistaxis. Evaluation of specimens by an experienced head and neck pathologist is crucial, as an imprecise histopathologic diagnosis may lead to adoption of an incorrect treatment strategy. In a series of 12 patients presenting to the University of Texas MD Anderson Cancer Center with a histopathologic diagnosis of esthesioneuroblastoma (ENB) from an outside institution, $10(83 \%)$ were considered to be misdiagnoses when specimens were reviewed. Of these patients, $8 / 10$ required significant alteration to a previously proposed treatment plan [27]. This underscores the importance of reviewing pathology slides obtained at outside institutions and one should have a low threshold to repeat a biopsy when a diagnosis is not confirmed.

High-resolution preoperative imaging is used to determine the extent of disease, the resectability of the lesion, and the surgical approach required to obtain a negative margin resection. Preoperative imaging includes high-resolution computed tomography (CT) and high-resolution skull base magnetic resonance imaging (MRI). High resolution MRI evaluation, performed with intravenous contrast, is critical in evaluating extent of disease and, when 
there is extension beyond the anterior skull base, the relationship of the mass to surrounding critical structures including the vasculature and cranial nerves [28]. A CT angiogram should be considered if there is concern for involvement of the carotid or vertebral arteries. A positron emission tomography (PET) scan is performed to assess for occult regional and metastatic disease. There are several staging systems currently used to guide management of these malignancies. The American Joint Committee on Cancer TNM staging system is most commonly used for most sinonasal malignancies [7]; separate staging systems have been developed for ENB by Kadish, Morita, and Dulguerov [29-31]. After thorough diagnostic workup, cases should be presented to a multidisciplinary head and neck tumor board for determination of the appropriate management plan for each patient on an individualized basis.

\section{Surgical Approaches}

After careful preoperative evaluation, a surgical approach is chosen as determined by histopathology, extent of disease, and surgeon skill and experience. Selection of approach is made with consideration of oncologic principles. Surgical approaches to anterior skull base lesions can be divided into traditional open craniofacial approaches, endoscopic-assisted approaches in which a craniotomy is combined with an endonasal endoscopic approach, and a purely endonasal endoscopic approach.

Expanded endonasal endoscopic approaches can be considered for sinonasal malignancies in the nasal cavity and paranasal sinuses including those with involvement of and extension through the anterior cranial base (Figs. 2, 3, 4). Contraindications for a purely endoscopic approach include significant intracranial, orbital, lateral maxillary, or palatal extension. Other contraindications include lateral extension above the orbit, involvement of the anterior table or lateral recesses of the frontal sinuses, involvement of the nasal bones, and extension into the soft tissues requiring removal of skin. In the absence of these contraindications, a completely endonasal endoscopic approach can be performed and may include the entirety of the anterior cranial base from the posterior table of the frontal sinus anteriorly to the planum sphenoidale posteriorly and bilaterally to the lamina papyraceae. It is important to note that the size of the tumor itself does not dictate the limit of the resection, but rather the histologic analysis of the tissue sent for intraoperative margin assessment. In other words, the tumor size is often smaller than the work volume permitted by an endoscopic endonasal approach. If margins cannot be cleared intraoperatively, conversion to an endoscopic-assisted or open approach should be considered contemporaneously or in a staged fashion and patients should be consented appropriately.
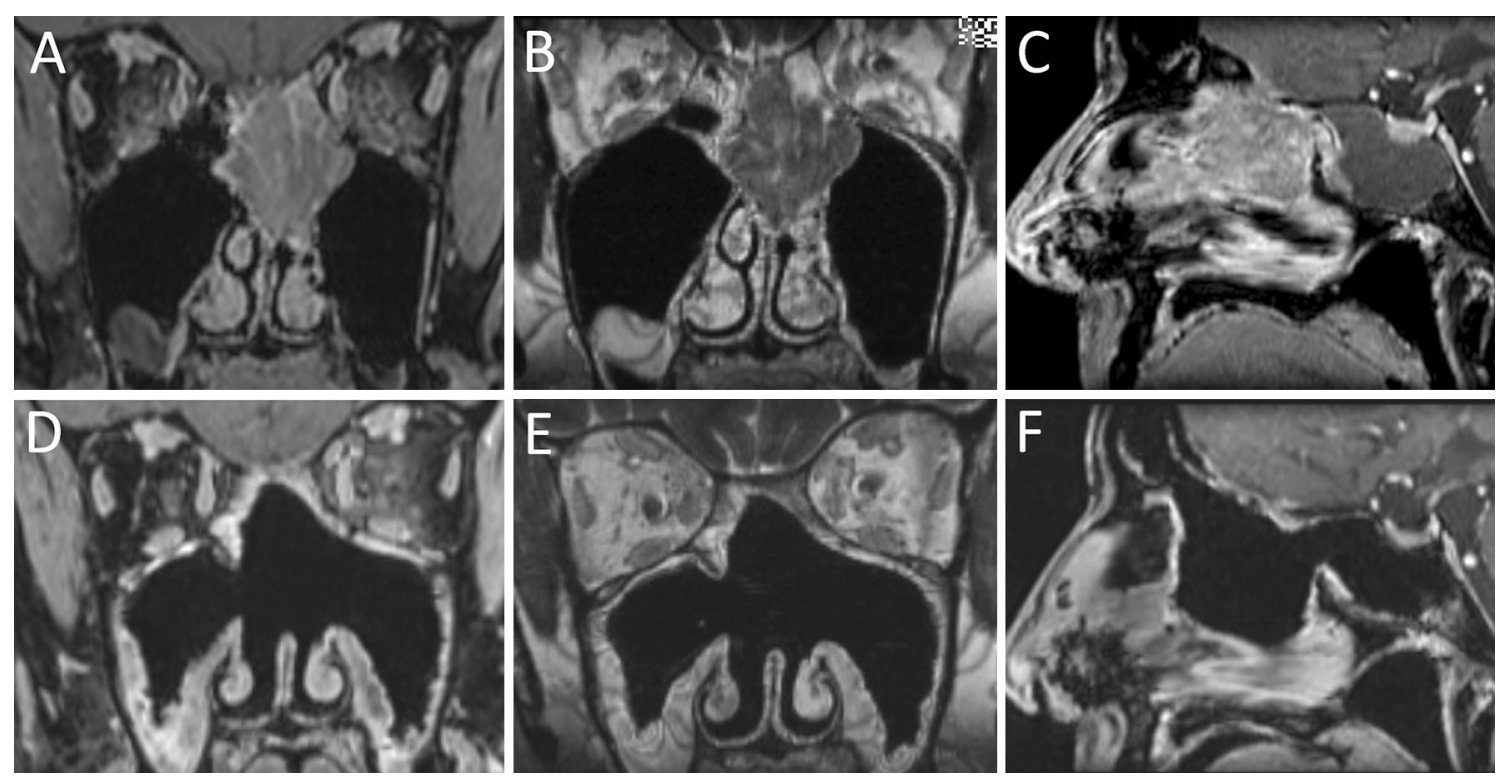

Fig. 2 Preoperative coronal $(\mathbf{a}, \mathbf{b})$ and sagittal (c) post contrast VIBE (a, c) and CISS (b) MRI sequences of a patient with a sinonasal undifferentiated carcinoma demonstrate an enhancing mass centered in the left nasal cavity spanning the nasal septum. There is extension into the sphenoid sinus and left maxillary sinus. There is extension superiorly to the skull base with focal extension across the left cribriform plate. The patient underwent an expanded endonasal endoscopic approach and resection. All intraoperative margins were negative for tumor. The patient received postoperative chemoradiotherapy. Coronal $(\mathbf{d}, \mathbf{e})$ and sagittal (f) post contrast $\operatorname{VIBE}(\mathbf{d}, \mathbf{f})$ and CISS (e) MRI sequences 26 months after surgery demonstrating no evidence of recurrent tumor 

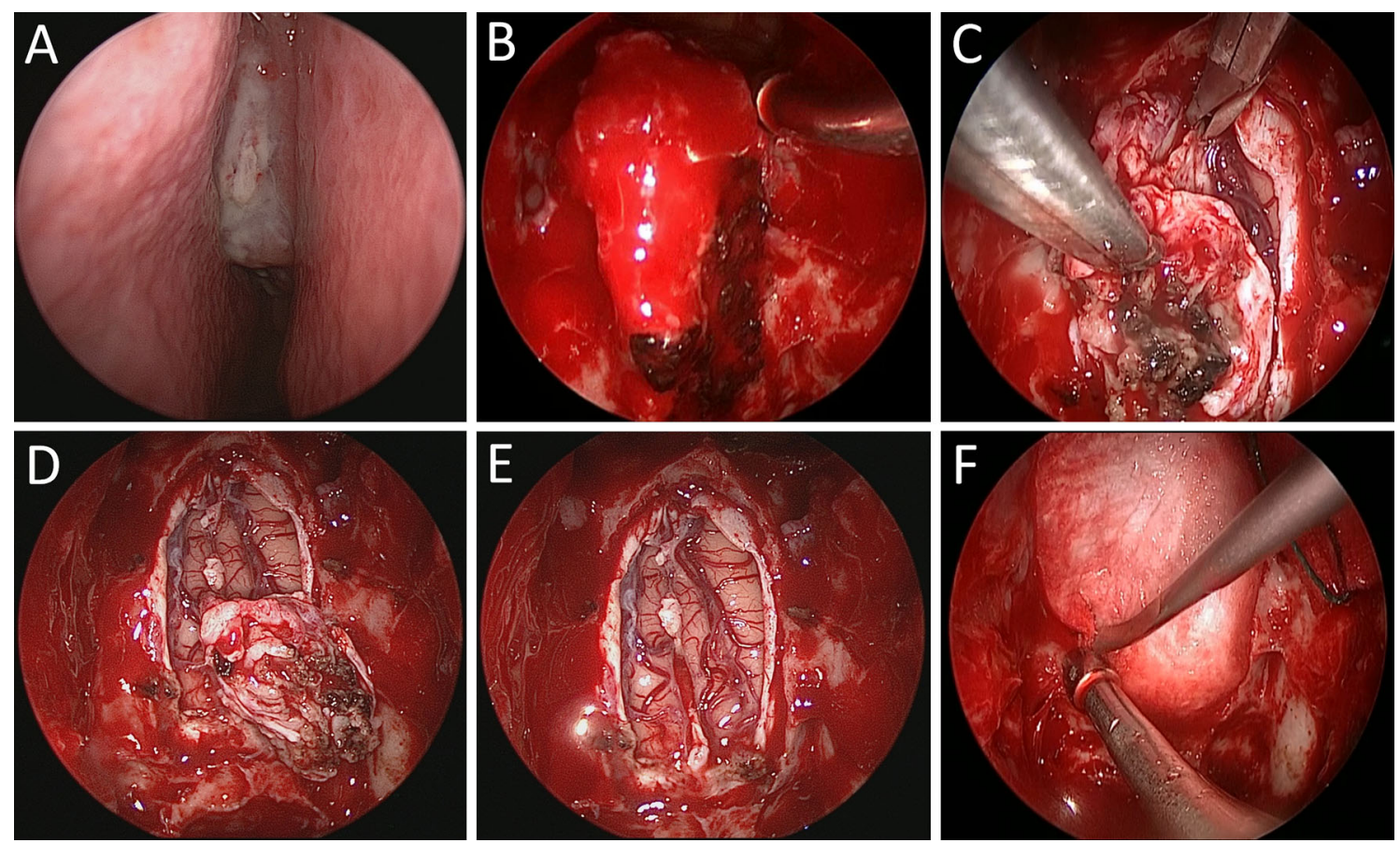

Fig. 3 Intraoperative photographs from the expanded endonasal endoscopic approach and resection performed on the patient in Fig. 2. a Initial view demonstrating a left sided nasal mass. Fibrinous material is noted over the previous outpatient biopsy site. b View after bilateral total sphenoethmoidectomies and modified Lothrop. The skull base is exposed circumferentially around the tumor which is seen centrally. Sinonasal and septal margins have been sent to pathology at this time. Extracranial margins have also been sent from the left ethmoids, planum sphenoidale, and right posterior ethmoids. Mucosa is still present in the right anterior ethmoid along the skull base. c View after coagulation and transection of the anterior and posterior ethmoidal arteries, skull base osteotomies, removal of the crista galli, and opening the dura sharply around the cribriform. Using a grasping forceps for counter traction, the dura extending along the left side of the crista is being cut with scissors. The left frontal lobe is

As noted previously, high resolution preoperative imaging is crucial to define the extent of the tumor to guide planning the optimal surgical approach. For tumors that extend posteriorly to involve the middle and/or posterior cranial fossae or laterally into the pterygopalatine and/or infratemporal fossae, additional surgical approaches can be added [24, 32-35]. In general, patients with disease extending laterally above the orbit, with significant intraorbital or intracranial involvement, and/or those with involvement of the nasal bones/facial soft tissues should be offered a traditional craniofacial resection rather than an expanded endonasal endoscopic approach (Fig. 5). For certain tumors that require a craniotomy to clear the superior, superolateral, and anterior aspects of disease but have defined nasal and paranasal sinus tumor that is resectable endoscopically, an endoscopic-assisted approach can be considered. also seen. d View after circumferential dural opening, transection of the falx, and cutting of the olfactory nerves. The tumor/cribriform specimen is seen in the right lower hand quadrant of the photograph with brain underneath. Margins were cut from this specimen on the back table. All sinonasal, extracranial skull base, and dural margins were negative. $\mathbf{e}$ View after removal of the specimen. The coagulated anterior and posterior ethmoidal arteries are visualized as well and the cut edge of the dura and falx. The bilateral frontal lobes are also seen. f View of one of the layers of reconstruction used being tucked between the dura and skull base. Not surprisingly, the nasal septum was involved with tumor in this case and a nasoseptal flap was unable to be used. The skull base was reconstructed with a Duragen inlay graft, a Duramatrix graft between the dura and bone (shown in panel), and a layer of Alloderm

\section{Endonasal Endoscopic Technique for Resection of Anterior Skull Base Malignancies}

Purely endonasal endoscopic approaches for resection of sinonasal malignancies have been described in the literature [36-41] and briefly outlined below. In most cases, a bilateral approach is performed and, for this reason, this is reviewed. After appropriate operating room set up and patient positioning, the endonasal approach begins with correcting access issues (e.g. septal deflection, etc.) and tumor debulking using a microdebrider. Care is taken to preserve normal tissue surrounding visible tumor boarders for subsequent intraoperative histopathological margin assessment. At this time, a nasoseptal flap may be raised in anticipation of the skull base reconstruction [42]; a nasoseptal flap can only be used if all intraoperative septal margins are negative. When permitted by extent of the 

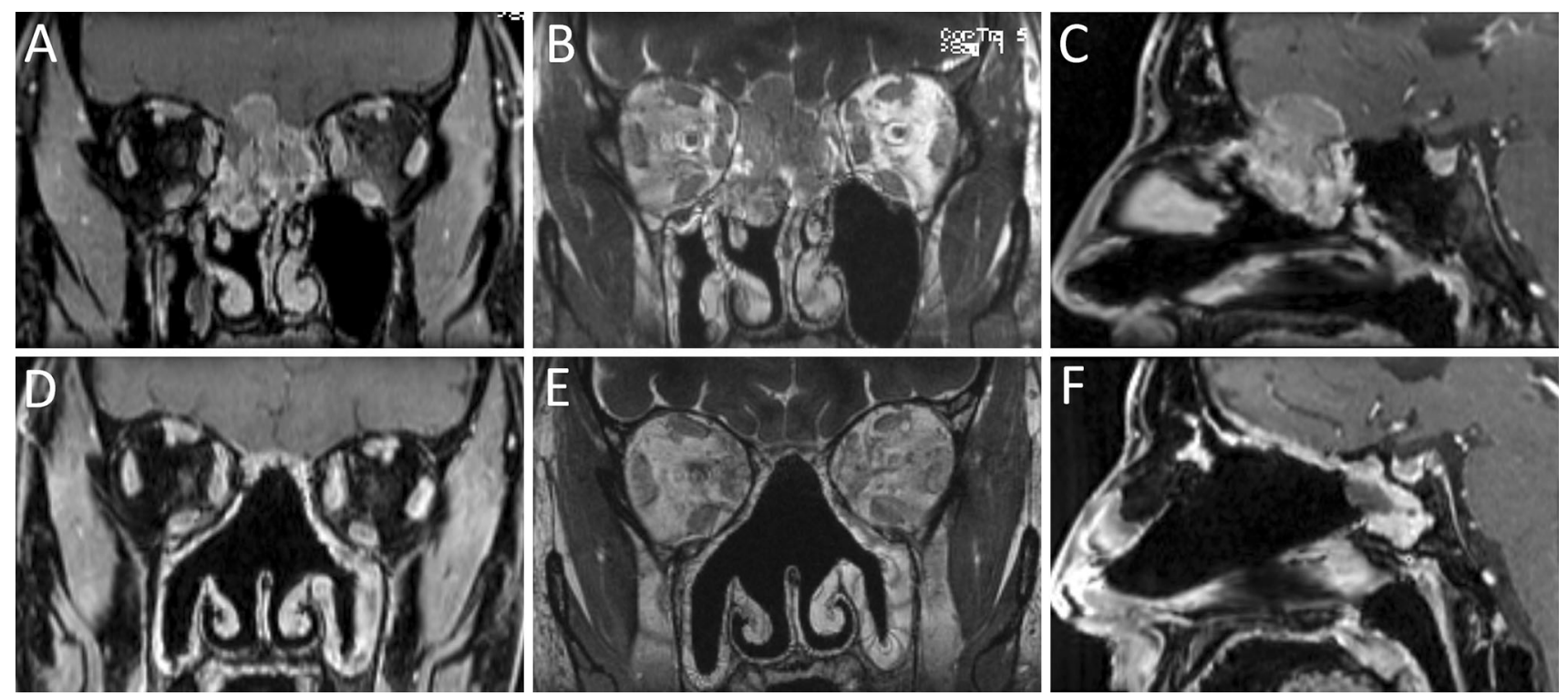

Fig. 4 High resolution preoperative coronal (a, b) and sagittal (c) post contrast VIBE $(\mathbf{a}, \mathbf{c})$ and $\operatorname{CISS}(\mathbf{b})$ MRI sequences of a patient with a Kadish stage D esthesioneuroblastoma demonstrate an enhancing sinonasal mass with intracranial extension. The lesion extends to the lamina papyraceae without evidence for intraorbital invasion. There is no evidence of brain invasion and there is a CSF cleft noted between the tumor and brain. On staging PET/CT, the patient also had FDG-positive right-sided neck adenopathy. The patient underwent an expanded endonasal endoscopic approach and resection. All intraoperative margins were negative. He also underwent a neck dissection, which confirmed neck disease. The patient received postoperative chemoradiotherapy. Coronal $(\mathbf{d}, \mathbf{e})$ and sagittal (f) post contrast VIBE $(\mathbf{d}, \mathbf{f})$ and CISS (e) MRI sequences 4 years after surgery demonstrating no evidence of recurrent tumor
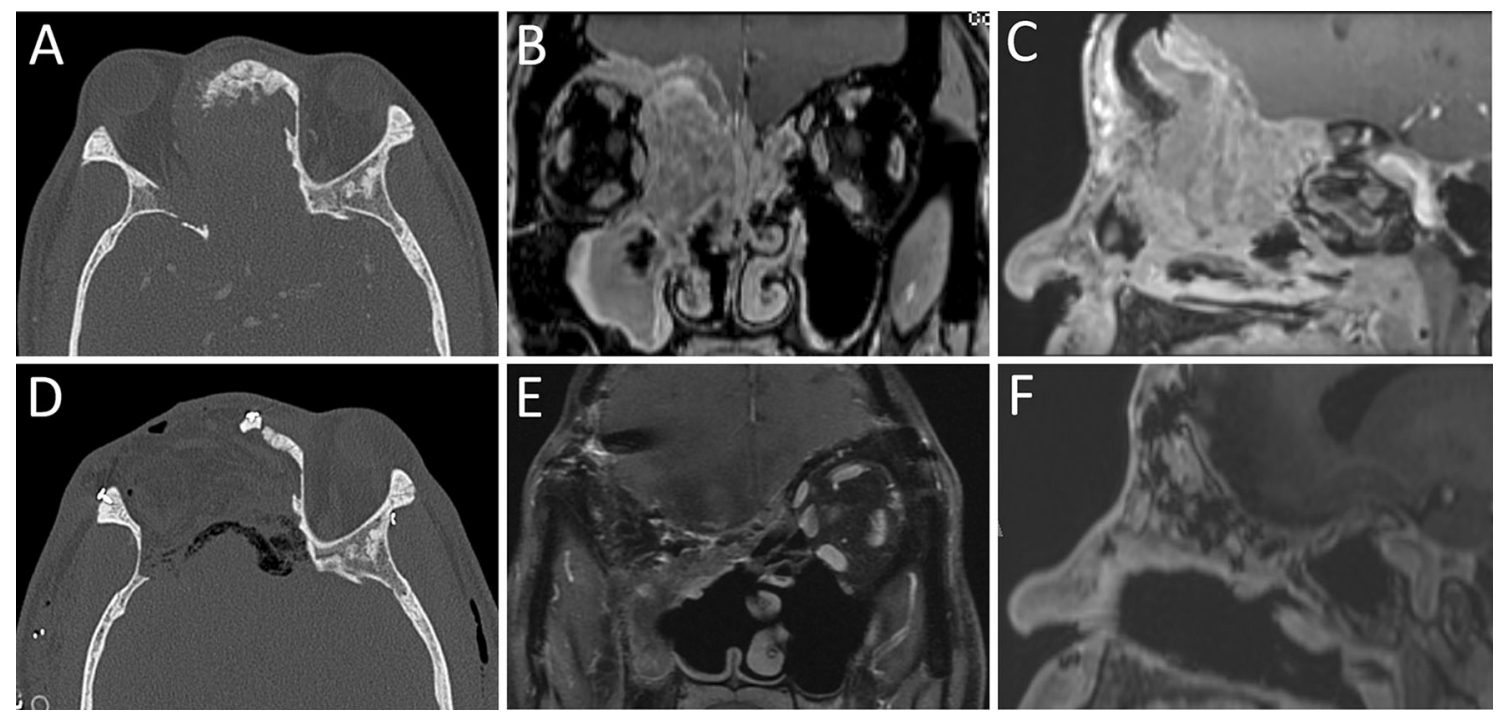

Fig. 5 Preoperative axial CT scan (a) and post-contrast coronal (b) and sagittal (c) VIBE MRI sequences of a patient with a poorly differentiated basaloid squamous cell carcinoma demonstrating an extensive tumor with the epicenter in the right ethmoid sinus. There is bony destruction of the right lamina papyracea with extension of the mass into the medial orbit. There is also bony destruction of the nasal bone, orbital roof, greater wing of the sphenoid, and floor of the anterior cranial fossa with intracranial extension of tumor and mass

tumor, surgery continues with bilateral middle turbinectomies, maxillary antrostomies, total ethmoidectomies, sphenoidotomies, and frontal sinus drill out (modified effect on the right inferior frontal lobe. The patient underwent an orbitocranial resection including an orbital exenteration. Reconstruction was performed with a radial forearm free flap. The patient underwent postoperative chemoradiotherapy. Postoperative axial CT (d) and post-contrast T1-weighted coronal (e) and sagittal (f) MRI sequences 16 months after surgery demonstrating no evidence of recurrent tumor. The free flap is seen nicely filling the orbit and under the anterior cranial base

Lothrop procedure). The rationale is to clear enough surrounding tissue to obtain precise circumferential contiguous margins as well as to create a wide corridor to the 

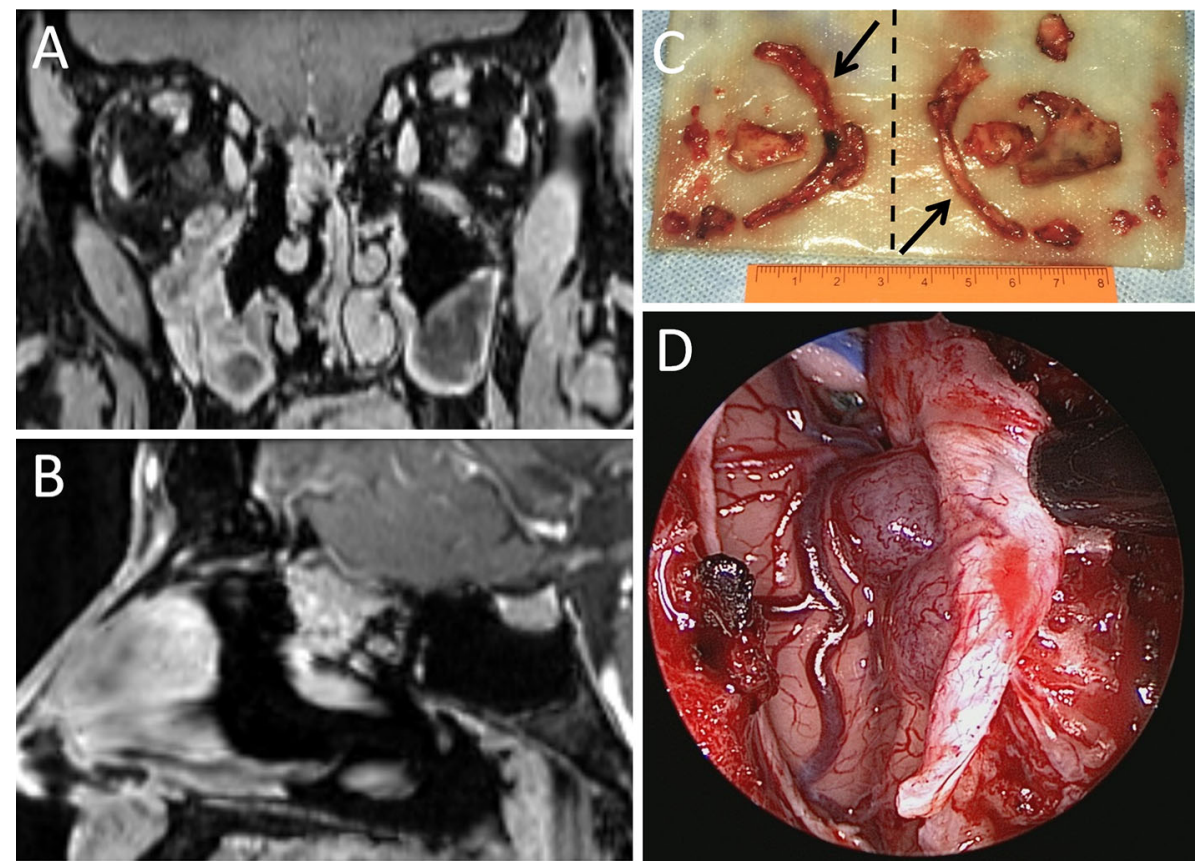

Fig. 6 Preoperative coronal (a) and sagittal (b) post contrast VIBE MRI sequences revealing a residual esthesioneuroblastoma at the right cribriform plate with intracranial extension (Kadish stage C). c Intraoperative photograph of the sinonasal margins sent during this procedure. The margins are displayed as in the surgical field; the right and left sided margins are on left and right sides of the vertical dotted line, respectively. The septal margins are noted by the black arrows; theses are typically divided into an anterior, middle and posterior septal margin when sent to pathology. d Intraoperative view following analysis of extracranial skull base margins, completion of

tumor. Septal incisions are then planned anteriorly, inferiorly, and posteriorly to clear the sagittal extent of tumor. When required, the sphenoid rostrum, sphenoid sinus septae, and posterior nasal septum are removed. At the end of this stage of the procedure, the entire anterior skull base from the posterior table of the frontal sinuses anteriorly to the sella turcica posteriorly and laterally from lamina papyracea to lamina papyracea is exposed (Fig. 3).

Prior to resection of the tumor and cribriform plate, precise contiguous circumferential margins are taken from the sinonasal cavity and extracranial surface of the skull base for frozen section analysis taking care to maintain proper orientation of each specimen (Fig. 6). The margin to visible tumor distance has not been defined for most anterior skull base malignancies, however, margins should be taken with sufficient distance from the tumor such that the probability of a positive intraoperative result is low while preserving as much structure as possible. Any bone involved with tumor or adjacent to positive mucosal margins is also removed. Areas of bone that cannot be resected are aggressively drilled. the skull base osteotomies, and opening the dura. Seen is the dural edge being reflected to the right of the photograph with purplish tumor underneath and attached along the posterior aspect to the right olfactory nerve. The right frontal lobe and coagulated right anterior ethmoidal artery are also seen. Once removed from the operative field, numerous dural margins were sent from this specimen as well as olfactory nerve margins. All intraoperative sinonasal, extracranial skull base, and dural and olfactory nerve margins were negative in this case

After circumferential margins are cleared, attention is turned to resection of the skull base and tumor. The anterior and posterior ethmoid arteries are controlled and osteotomies are performed lateral to the cribriform. Next, anterior and posterior osteotomies are created based on the planned dural cuts. These osteotomies are subsequently connected to the lateral osteotomies. The crista galli is then dissected from the surrounding dura, drilled at the attachment to the posterior table, and then removed, effectively separating the cribriform plate from the surrounding skull base (Fig. 3).

Next, circumferential dural cuts are performed, distal to the margins of the tumor. The falx cerebri is cut and arachnoid adhesions are also incised. The olfactory nerves are incised. The cribriform and tumor specimen is subsequently removed through the nasal cavity (Fig. 3). Once the specimen is removed, precise contiguous dural margins are cut from the specimen; olfactory nerve margins are also sent. Additional endoscopic resection is performed if needed to clear any positive margins. Attention is then turned to reconstruction of the skull base. 


\section{Skull Base Reconstruction}

The overall goal of skull base reconstruction is separation of the intracranial and sinonasal compartments to prevent cerebrospinal fluid (CSF) leak and its sequelae including pneumocephalus and meningitis. Principles of skull base reconstruction mandate a multi-layered watertight closure. There are numerous techniques and materials available for reconstruction of the skull base. In cases of large defects and/or high flow intraoperative CSF leaks, vascularized local pedicled flaps, such as the nasoseptal flap [42], are the gold standard. In cases in which tumor involvement precludes use of the nasoseptal flap, other pedicled flaps, autografts, or allografts can successfully be used [43-50]. Complete discussion of all reconstructive options is beyond the scope of this paper and is reviewed elsewhere [51-55].

\section{Surgical Management of the Neck and Orbit}

In cases in which there is radiologic evidence of disease in the neck, gross cervical lymphadenopathy, or a high probability of locoregional metastasis based on tumor stage, the neck must be included in the treatment algorithm. Treatment may consist of concomitant neck dissection and/or postoperative radiotherapy. Management of orbital disease is controversial. Previously, the standard of care was orbital exenteration. Eye-sparing protocols, however, have been developed that involve radiation or chemotherapy, which may be employed in cases where the tumor does not violate the periorbita [56]. In cases of extension into the orbit, endoscopic approaches with curative intent and eye conservation have more recently been employed. In a series of 21 malignant tumors managed endoscopically, 6 (28.6\%) were noted to have a recurrence [57]. These results underscore the difficulty of managing orbital disease in this patient population.

\section{Importance of Frozen Section Analysis in Surgical Resection of Sinonasal Malignancies}

Traditional oncologic dogma has espoused an en bloc resection as tantamount to achieving a complete resection. Consequently, there was significant controversy as to whether endoscopic techniques, which involve piecemeal tumor resection, could be successfully employed in this patient population with sound oncologic outcomes. Due to the complexities of the skull base, en bloc resections are challenging, regardless of the surgical approach utilized and data supports margin status as being the most important prognosticator of survival rather than the manner in which the tumor was removed [37, 58-60]. As such, frozen section margins are essential to ensure complete resection of all microscopic disease. Thus, the surgical team relies heavily on the expertise of the surgical pathologist intraoperatively. Anterior skull base malignancies occupy a complex three-dimensional anatomic region that results in multiple intraoperative frozen section margins (Fig. 6). Due to the number of frozen section margins required, the chance of a false negative frozen section is not trivial. In a study of 68 patients undergoing endoscopic endonasal resection of sinonasal malignancies, Manjunath et al. [61] noted a mean of 10.8 margins per case and found a false negative rate of $22.1 \%$. Such false negative reads on frozen section analysis impact the management algorithm and options in these situations include return to operating room for re-resection with additional surgical and anesthetic morbidity or alteration of adjuvant therapy (i.e. addition of chemotherapy) along with its associated morbidity.

\section{Postoperative Care}

The postoperative protocol at the Johns Hopkins Hospital includes overnight monitoring in the neuroscience intensive care unit. A head CT is often performed the night of surgery to evaluate for bleeding, pneumocephalus, as well as the position of the reconstruction and nasal packing. Transfer to the surgical floor generally occurs on postoperative day 1 and an MRI scan is performed within $48 \mathrm{~h}$ of the surgery to assess extent of resection. Post-operative MRI performed within $48 \mathrm{~h}$ allows for evaluation of extent of resection prior to formation of most enhancing granulation tissue and is useful to establish a new post-operative baseline for comparison. When required, lumbar drainage is continued for $36-48 \mathrm{~h}$ postoperatively. Regionally appropriate intravenous antibiotics are continued throughout the hospital stay and the patient is discharged on oral antibiotics until the nasal packing is removed.

Patients are followed after discharge with serial nasal endoscopy to assess the integrity of the skull base. Conservative nasal debridement may be performed, but crusting along the skull base is not disturbed so as not to disrupt the reconstruction. Once endoscopic examination confirms an intact skull base and the nasal packing has begun to pull away from the reconstruction site, the patient may begin nasal saline irrigations. Adjuvant radiation therapy is generally started 6-8 weeks postoperatively. Adjuvant chemotherapy may be added as indicated by histopathological diagnosis, stage of tumor, and extent of surgical resection. Sinonasal irrigations and regular in-office debridements are conducted in order to limit the crusting in the area of the reconstruction that may occur during radiation. 
Postoperative surveillance includes regular physical examination with nasal endoscopy and thorough head and neck examination to assess for evidence of gross tumor recurrence or locoregional metastases. In general, followup MRI scans are performed at 3-4 month intervals for the first year postoperatively, 6-month intervals during the next couple of years, and yearly thereafter. Timing of PET/CT scanning is determined by histopathologic diagnosis and stage of disease at presentation.

\section{Complications of Surgical Approaches to Resection of Sinonasal Malignancies}

One of the most common complications of expanded endonasal approaches in the management of sinonasal and anterior skull base malignancies is CSF leak, with an incidence of $<5 \%$ in centers with significant endoscopic experience and expertise $[62,63]$. Other complications that should be considered include meningitis, pneumocephalus, neurovascular complications, and ocular complications including visual loss. Traditional open craniofacial approaches have been shown to have an overall complication rate around $30 \%$ in a large international collaborative cohort [64] although some more contemporary open craniofacial series report significantly less morbidity [65]. Expanded endonasal endoscopic approaches are associated with low morbidity and in two large endoscopic series of sinonasal malignancies, the overall complication rate was $9-11 \%[62,63]$.

\section{Outcomes}

There has been an increasing number of studies published reporting outcomes of patients with sinonasal malignancies treated using endoscopic surgical techniques. This literature was extensively reviewed by Lund et al. [5]. The diverse histopathology, small number of patients, variability in stage, and shorter-term follow-up in many of these studies provide challenges in evaluating the impact of endoscopic surgical approaches on disease-specific and overall survival. This is an active area of interest and ongoing analysis.

Two of the largest series evaluating outcomes of patients treated with endoscopic techniques were published in the late 2000s. In a 10-year experience reported by Nicolai et al. [62] on 134 patients undergoing an expanded endonasal approach, the 5-year disease-specific survival was $91 \%$ compared to $59 \%$ in a group of 50 patients undergoing a cranioendoscopic approach. An independent study of 120 patients by Hanna et al. [63] showed a 5-year disease-specific survival of $87 \%$ overall with no significant differences in disease recurrence and survival in patients who underwent an exclusively endoscopic approach $(n=93)$ compared to those who underwent a cranioendoscopic approach $(n=27)$. These two studies provide evidence that in experienced hands, endonasal endoscopic surgery can be considered an oncologically sound approach to sinonasal malignancies. Moreover, a recent systematic review comparing endoscopic versus open craniofacial resection techniques reported no statistically significant difference in 5-year overall and diseasespecific survival or locoregional control rates in patients with low-stage malignancies [66]. Thus, in properly selected patients, good oncologic outcomes can be achieved using this approach in conjunction with appropriate adjuvant therapy in the setting of a multidisciplinary management team.

\section{Conclusion}

Sinonasal malignancies are rare tumors that represent a wide variety of histopathologic diagnoses. These tumors generally present at an advanced stage due to non-specific symptoms that often lead to a delayed diagnosis. Advanced disease stage, combined with the complex anatomy of the sinonasal cavities and anterior skull base have traditionally rendered management of these tumors difficult. In appropriately selected patients, these tumors are increasingly being managed successfully with endoscopic surgical approaches with decreased morbidity and mortality. Although the limits of endoscopic surgery in the treatment of patients with sinonasal malignancies have yet to be defined and longer follow-up with a larger number of patients is required to further assess the role of endonasal endoscopic surgery, these techniques certainly have an important role in the management of patients with sinonasal and anterior skull base malignancies and are essential for contemporary skull base centers of excellence.

\section{References}

1. Dutta R, Dubal PM, Svider PF, et al. Sinonasal malignancies: a population-based analysis of site-specific incidence and survival. Laryngoscope. 2015;125:2491-7.

2. Weber AL, Stanton AC. Malignant tumors of the paranasal sinuses: radiologic, clinical, and histopathologic evaluation of 200 cases. Head Neck Surg. 1984;6:761-76.

3. Barnes L, Eveson JW, Reichart P, Sidransky D, editors. World Health Organization classification of tumours: pathology and genetics of head and neck tumours. Lyon: IARC Press; 2005. p. 10. 
4. Dulguerov P, Jacobsen MS, Allal AS, et al. Nasal and paranasal sinus carcinoma: are we making progress? A series of 220 patients and a systematic review. Cancer. 2001;92:3012-29.

5. Lund VJ, Stammberger H, Nicolai P, et al. European position paper on endoscopic management of tumours of the nose, paranasal sinuses, and skull base. Rhinol Suppl. 2010;22:1-143.

6. Banuchi V, Mallen J, Kraus D. Cancers of the nose, sinus, and skull base. Surg Oncol Clin N Am. 2015;24:563-77.

7. National Comprehensive Cancer Network. Head and Neck Cancers. Version 1.2015. www.nccn.org/professionals/physician_gls/ pdf/head-and-neck.pdf. Accessed 15 Aug 2015.

8. Ketcham AS, Wilkins RH, Van Buren JM, et al. A combined intracranial facial approach to the paranasal sinuses. Am J Surg. 1963;106:698-703.

9. Yuen AP, Fung CF, Hung KN. Endoscopic cranionasal resection of anterior skull base tumor. Am J Otolaryngol. 1997;18:431-3.

10. Anderhuber W, Stammberger H, Walch C, et al. Rigid endoscopy in minimally invasive therapy of tumours of the paranasal sinuses and skull base. Minim Invasive Ther Allied Technol. 1999;8:25-32.

11. Stammberger H, Anderhuber W, Walch C, Papaefthymiou G. Possibilities and limitations of endoscopic management of nasal and paranasal sinus malignancies. Acta Otorhinolaryngol Belg. 1999;53:199-205.

12. Thaler ER, Kotapka M, Lanza DC, Kennedy DW. Endoscopically assisted anterior cranial skull base resection of sinonasal tumors. Am J Rhinol. 1999;13:303-10.

13. Walch C, Stammberger H, Anderhuber W, et al. The minimally invasive approach to olfactory neuroblastoma: combined endoscopic and stereotactic treatment. Laryngoscope. 2000;110:635-40.

14. Goffart Y, Jorissen M, Daele J, et al. Minimally invasive endoscopic management of malignant sinonasal tumours. Acta Otorhinolaryngol Belg. 2000;54:221-32.

15. Locatelli D, Castelnuovo P, Santi L, et al. Endoscopic approaches to the cranial base: perspectives and realities. Childs Nerv Syst. 2000;16:686-91.

16. Unger F, Walch C, Stammberger H, et al. Olfactory neuroblastoma (esthesioneuroblastoma): report of six case treated by a novel combination of endoscopic surgery and radiosurgery. Minim Invasive Neurosurg. 2001;44:79-84.

17. Casiano RR, Numa WA, Falquez AM. Endoscopic resection of esthesioneuroblastoma. Am J Rhinol. 2003;17:185-90.

18. Roh HJ, Batra PS, Citardi MJ, et al. Resection of sinonasal malignancies: a preliminary report. Am J Rhinol. 2004;18:239-46.

19. Shipchandler TZ, Batra PS, Citardi MJ, et al. Outcomes for endoscopic resection of sinonasal squamous cell carcinoma. Laryngoscope. 2005;115:1983-7.

20. Unger F, Haselsberger K, Walch C, et al. Combined endoscopic surgery and radiosurgery as treatment modality for olfactory neuroblastoma (esthesioneuroblastoma). Acta Neurochir. 2005;147:595-601.

21. Batra PS, Citardi MJ, Worley S, et al. Skull base tumors: comparison of combined traditional and endoscopic techniques. Am J Rhinol. 2005;19:521-8.

22. Castelnuovo P, Dallan I, Battaglia P, et al. Endoscopic skull base surgery: past, present and future. Eur Arch Otorhinolaryngol. 2010;267:649-63.

23. Bhatki AM, Carrau RL, Snyderman CH, et al. Endonasal surgery of the ventral skull base-endoscopic transcranial surgery. Oral Maxillofac Surg Clin North Am. 2010;22:157-68.

24. Kassam AB, Prevedello DM, Carrau RL, et al. Endoscopic endonasal skull base surgery: analysis of complications in the authors' initial 800 patients. J Neurosurg. 2011;114:1544-68.

25. Kasemsiri P, Carrau RL, Ditzel Filho LF, et al. Advantages and limitations of endoscopic endonasal approaches to the skull base. World Neurosurg. 2014;82(6 Suppl):S12-21.
26. DeMonte F. Evolving role of skull base surgery for patients with low and high grade malignancies. J Neurooncol. 2004;69:191-8.

27. Cohen ZR, Marmor E, Fuller GN, et al. Misdiagnosis of olfactory neuroblastoma. Neurosurg Focus. 2002;12:1-6.

28. Blitz AM, Choudhri AF, Chonka ZD, et al. Anatomic considerations, nomenclature, and advanced cross-sectional imaging techniques for visualization of the cranial nerve segments by MR imaging. Neuroimaging Clin N Am. 2014;24:1-15.

29. Kadish S, Goodman M, Wang CC. Olfactory neuroblastoma: a clinical analysis of 17 cases. Cancer. 1976;37:1571-6.

30. Morita A, Ebersold MJ, Olsen KD, et al. Esthesioneuroblastoma: prognosis and management. Neurosurgery. 1993;32:706-14.

31. Dulguerov P, Calcaterra T. Esthesioneuroblastoma: the UCLA experience 1970-1990. Laryngoscope. 1992;102:843-9.

32. Kassam AB, Gardner P, Snyderman C, Mintz A, Carrau R. Expanded endonasal approach: fully endoscopic, completely transnasal approach to the middle third of the clivus, petrous bone, middle cranial fossa, and infratemporal fossa. Neurosurg Focus. 2005;19:E6.

33. Kassam A, Snyderman CH, Mintz A, Gardner P, Carrau RL. Expanded endonasal approach: the rostrocaudal axis. Part II. Posterior clinoids to the foramen magnum. Neurosurg Focus. 2005;19:E4.

34. Cavallo LM, Messina A, Cappabianca P, et al. Endoscopic endonasal sugery of the midline skull base: anatomical study and clinical considerations. Neurosurg Focus. 2005;19:E2.

35. Cavallo LM, Messina A, Gardner P, et al. Extended endoscopic endonasal approach to the pterygopalatine fossa: anatomical study and clinical considerations. Neurosurg Focus. 2005;19:E5.

36. Carrau RL, Kassam AB, Snyderman CH, et al. Endoscopic transnasal anterior skull base resection for the treatment of sinonasal malignancies. Oper Tech Otolaryngol. 2006;17:102-10.

37. Snyderman CH, Carrau RL, Kassam AB, et al. Endoscopic skull base surgery: principles of oncological surgery. J Surg Oncol. 2008;97:658-64.

38. Nicolai P, Castelnuovo P, Villaret AB. Endoscopic resection of sinonasal malignancies. Curr Oncol Rep. 2011;13:138-44.

39. Gallia GL, Reh DD, Salmasi V, et al. Endonasal endoscopic resection of esthesioneuroblastoma: the Johns Hopkins experience and review of the literature. Neurosurg Rev. 2011;34:465-75.

40. Kasemsiri P, Prevedello DM, Otto BM, et al. Endoscopic endonasal technique: treatment of paranasal and anterior skull base malignancies. Braz J Otorhinolaryngol. 2013;79:760-9.

41. Castelnuovo P, Battaglia P, Turri-Zanoni M, et al. Endoscopic endonasal surgery for malignancies of the anterior cranial base. World Neurosurg. 2014;82(6 Suppl):S22-31.

42. Hadad G, Bassagasteguy L, et al. A novel reconstructive technique after endoscopic expanded endonasal approaches: vascular pedicle nasoseptal flap. Laryngoscope. 2006;116:1882-6.

43. Fortes FS, Carrau RL, Snyderman CH, et al. Transpterygoid transposition of a temporoparietal fascia flap: a new method for skull base reconstruction after endoscopic expanded endonasal approaches. Laryngoscope. 2007;117:970-6.

44. Fortes FS, Carrau RL, Snyderman CH, et al. The posterior pedicle inferior turbinate flap: a new vascularized flap for skull base reconstruction. Laryngoscope. 2007;117:1329-32.

45. Prevedello DM, Barges-Coll J, Fernandez-Miranda JC, et al. Middle turbinate flap for skull base reconstruction: cadaveric feasibility study. Laryngoscope. 2008;119:2094-8.

46. Zanation AM, Snyderman CH, Carrau RL, et al. Minimally invasive endoscopic pericranial flap: a new method for endonasal skull base reconstruction. Laryngoscope. 2009;119:13-8.

47. Luginbuhl AJ, Campbell PG, Evans J, et al. Endoscopic repair of high-flow cranial base defects using a bilayer button. Laryngoscope. 2010;120:876-80. 
48. Illing E, Chaaban MR, Riley KO, et al. Porcine small intestine submucosal graft for endoscopic skull base reconstruction. Int Forum Allergy Rhinol. 2013;3:928-32.

49. Germani RM, Vivero R, Herzallah IR, et al. Endoscopic reconstruction of large anterior skull base defects using acellular dermal allograft. Am J Rhinol. 2007;21:615-8.

50. Lorenz RR, Dean RL, Hurley DB, et al. Endoscopic reconstruction of anterior and middle cranial fossa defects using acellular dermal allograft. Laryngoscope. 2003;113:496-501.

51. Kassam A, Carrau RL, Snyderman $\mathrm{CH}$, et al. Evolution of reconstructive techniques following endoscopic expanded endonasal approaches. Neurosurg Focus. 2005;19:E8.

52. Esposito F, Dusick JR, Fatemi N, et al. Graded repair of cranial base defects and cerebrospinal fluid leaks in transsphenoidal surgery. Neurosurgery. 2007;60(4 Suppl 2):295-303.

53. Tabaee A, Anand VK, Brown SM, et al. Algorithm for reconstruction after endoscopic pituitary and skull base surgery. Laryngoscope. 2007;117:1133-7.

54. Harvey RJ, Parmar P, Sacks R, et al. Endoscopic skull base reconstruction of large dural defects: a systematic review of published evidence. Laryngoscope. 2012;122:452-9.

55. Soudry E, Turner JH, Nayak JV, et al. Endoscopic reconstruction of surgically created skull base defects: a systematic review. Otolaryngol Head Neck Surg. 2014;150:730-8.

56. Perry C, Levine PA, Williamson BR, et al. Preservation of the eye in paranasal sinus cancer surgery. Arch Otolaryngol Head Neck Surg. 1988;114:632-4.

57. Christianson B, Perez C, Harrow B, Batra PS. Management of the orbit during endoscopic sinonasal tumor surgery. Int Forum Allergy Rhinol. 2015;5:967-73.
59. Patel SG, Singh B, Polluri A, et al. Craniofacial surgery for malignant skull base tumors: report of an international collaborative study. Cancer. 2003;98:1179-87.

60. Bentz BG, Bilsky MH, Shah JP, et al. Anterior skull base surgery for malignant tumors: a multivariate analysis of 27 years of experience. Head Neck. 2003;25:515-20.

61. Manjunath L, Derousseau T, Batra PS. Prognostic value of surgical margins during endoscopic resection of paranasal sinus malignancy. Int Forum Allergy Rhinol. 2015;5:454-9.

62. Nicolai P, Battaglia P, Bignami M, et al. Endoscopic surgery for tumors of the sinonasal tract and adjacent skull base: a 10 year experience. Am J Rhinol. 2008;22:308-16.

63. Hanna E, DeMonte F, Ibrahim S, et al. Endoscopic resection of sinonasal cancers with and without craniotomy: oncologic results. Arch Otolaryngol Head Neck Surg. 2009;135:1219-34.

64. Ganly I, Patel SG, Singh B, et al. Craniofacial resection for malignant paranasal sinus tumors: report of an international collaborative study. Head Neck. 2005;27:575-84.

65. Raza SM, Garzon-Muvdi T, Gallia GL, et al. Craniofacial resection of midline anterior skull base malignancies: a reassessment of outcomes in the modern era. World Neurosurg. 2011;78:128-36.

66. Higgins TS, Thorp B, Rawlings BA, et al. Outcome results of endoscopic vs craniofacial resection of sinonasal malignancies: a systematic review and pooled-data analysis. Int Forum Allergy Rhinol. 2011;1:255-61. 